PROCEEDINGS OF THE

AMERICAN MATHEMATICAL SOCIETY

Volume 137, Number 1, January 2009, Pages 353-357

S 0002-9939(08)09598-1

Article electronically published on August 6, 2008

\title{
BOUNDING MATRIX COEFFICIENTS FOR SMOOTH VECTORS OF TEMPERED REPRESENTATIONS
}

\author{
BINYONG SUN
}

(Communicated by Wen-Ching Winnie Li)

\begin{abstract}
Let $G$ be a Lie group. Let $(\pi, V)$ be a unitary representation of $G$ which is weakly contained in the regular representation. For smooth vectors $u, v$ in $V$, we give an upper bound for the matrix coefficient $\langle\pi(g) u, v\rangle$, in terms of Harish-Chandra's $\Xi$-function.
\end{abstract}

\section{INTRODUCTION}

Let $G$ be a locally compact Hausdorff topological group, with a compact subgroup $K$ and a closed subgroup $P$ such that $G=K P$.

Denote by $\Delta_{G}$ the modular function of $G$, which is defined by

$$
\int_{G} f(g x) d g=\Delta_{G}^{-1}(x) \int_{G} f(g) d g, \quad \text { for all } f \in \mathrm{L}^{1}(G ; d g), x \in G,
$$

where " $d g$ " is a left invariant Haar measure on $G$. Denote by $\Delta_{P}$ the modular function of $P$, and write

$$
\delta(p)=\frac{\Delta_{G}(p)}{\Delta_{P}(p)}, \quad p \in P .
$$

Extend $\delta$ to a left $K$-invariant function on $G$, which is still denoted by $\delta$, by the formula

$$
\delta(k p)=\delta(p), \quad k \in K, p \in P .
$$

Under this general setting, Harish-Chandra's basic spherical function $\Xi$ is still defined by

$$
\Xi(g)=\Xi_{K, P}(g)=\int_{K} \delta^{-\frac{1}{2}}(g k) d k, \quad g \in G,
$$

where " $d k$ " is the normalized Haar measure on $K$.

Let $(\pi, V)$ be a unitary representation of $G$. For any $v \in V$, write

$$
\|v\|=\sqrt{\langle v, v\rangle}
$$

and

$$
d_{v}=\text { the dimension of the space spanned by } \pi(K) v \text {. }
$$

The following is a formal generalization of a fundamental result of Cowling, Haagerup and Howe [1, Theorem 2]. Although in [1, the theorem is only stated for

Received by the editors September 7, 2007.

2000 Mathematics Subject Classification. Primary 22E45; Secondary 22E30.

This work was supported by the Knowledge Innovation Program of the Chinese Academy of Sciences.

(C)2008 American Mathematical Society Reverts to public domain 28 years from publication 
$G$ semisimple algebraic, and $G=K P$ an Iwasawa decomposition, the same proof works in our general setting. So we omit the proof.

Theorem 1.1. If $\pi$ is weakly contained in the regular representation, and $u, v$ are $K$-finite vectors in $V$, then

$$
|\langle\pi(g) u, v\rangle| \leq\left(d_{u} d_{v}\right)^{\frac{1}{2}}\|u\|\|\mid v\| \Xi(g)
$$

for all $g \in G$.

For the notion of weak containment, see [1, Page 98]. The left regular representation is canonically isomorphic to the right regular one. So we do not distinguish them and call any of them the regular representation. A unitary representation which is weakly contained in the regular representation is also called tempered. Notice that $\Xi$ is the diagonal matrix coefficient for a $K$-fixed vector in $\operatorname{Ind}_{P}^{G} 1_{P}$. Here we use normalized induction, and $1_{P}$ denotes the trivial representation of $P$. When $P$ is amenable, i.e., when $1_{P}$ is tempered, the unitary representation $\operatorname{Ind}_{P}^{G} 1_{P}$ is also tempered. Recall that $P$ is amenable in the most interesting case, i.e., when $G$ is reductive algebraic over a local field and $P$ is a minimal parabolic subgroup of it.

In applications, in particular for branching problems, one usually needs the bound (1.1) to be valid for smooth vectors. When $G$ is totally disconnected, $K$ is usually chosen to be open. Then smooth vectors are the same as $K$-finite ones. But when $G$ is a Lie group, in most cases, there are more smooth vectors than $K$-finite ones.

From now on assume that $G$ is a Lie group, with complexified Lie algebra $\mathfrak{g}_{\mathbb{C}}$. Let "U" stand for the universal enveloping algebra. Still denote by $\pi$ the induced action of $\mathrm{U}\left(\mathfrak{g}_{\mathbb{C}}\right)$ on the smooth vectors $V^{\infty}$ of $V$. Recall that $V^{\infty}$ is a Fréchet space under the seminorms

$$
|v|_{X}=\|\pi(X) v\|, \quad v \in V^{\infty},
$$

where $X$ runs through all vectors in $\mathrm{U}\left(\mathfrak{g}_{\mathbb{C}}\right)$.

Denote by $\mathfrak{k}$ the Lie algebra of $K$, with complexification $\mathfrak{k}_{\mathbb{C}}$, and denote by $\mathrm{U}\left(\mathfrak{k}_{\mathbb{C}}\right)^{K}$ the centralizer of $K$ in $\mathrm{U}\left(\mathfrak{k}_{\mathbb{C}}\right)$.

Theorem 1.2. There is an element $X \in \mathrm{U}\left(\mathfrak{k}_{\mathbb{C}}\right)^{K}$, which depends on $K$ only, such that if $\pi$ is weakly contained in the regular representation, then

$$
|\langle\pi(g) u, v\rangle| \leq|u|_{X}|v|_{X} \Xi(g)
$$

for all $u, v \in V^{\infty}$ and $g \in G$, and

$$
|\langle\pi(g) u, v\rangle| \leq d_{v}^{\frac{1}{2}}|u|_{X}\|v\| \Xi(g)
$$

for all $v \in V$ that are $K$-finite, $u \in V^{\infty}$, and $g \in G$.

In the case that $G$ is real reductive, $K$ is a maximal compact subgroup, and $P$ is a minimal parabolic subgroup, Wallach has an estimate that (3, Proposition $5.1 .2])$

$$
\left|\lambda\left(\pi_{K}(g) u\right)\right| \leq \sigma(u)(1+\log || g||)^{d} \Xi(g), \quad u \in V_{K}^{\infty}, g \in G,
$$

where $\left(\pi_{K}, V_{K}^{\infty}\right)$ is the Casselman-Wallach smooth globalization of a tempered Harish-Chandra $\left(\mathfrak{g}_{\mathbb{C}}, K\right)$-module, $\|g\|$ is a certain "norm function" on $G, d$ is a nonnegative number which depends on $V_{K}^{\infty}, \lambda$ is a $K$-finite continuous linear functional on $V_{K}^{\infty}$, and $\sigma$ is a continuous seminorm on $V_{K}^{\infty}$ which depends on $\lambda$. Recall 
that a Harish-Chandra $\left(\mathfrak{g}_{\mathbb{C}}, K\right)$-module is tempered if and only if all of its irreducible subquotients globalize to tempered unitary representations. Our estimate (1.3) improves (1.4) in the unitary case.

The following is a direct consequence of (1.2), which is a base for applying matrix coefficient integrals to branching problems.

Corollary 1.3. Let $H$ be a closed unimodular subgroup of $G$, with a fixed Haar measure dh. If $\pi$ is weakly contained in the regular representation, and $\left.\Xi\right|_{H} \in$ $\mathrm{L}^{1}(H)$, then

$$
\begin{aligned}
V^{\infty} \times V^{\infty} & \rightarrow \mathbb{C}, \\
u, v & \mapsto \int_{H}\langle\pi(h) u, v\rangle d h
\end{aligned}
$$

is a well-defined continuous $(H \times H)$-invariant Hermitian form on $V^{\infty}$.

The condition that $\left.\Xi\right|_{H} \in \mathrm{L}^{1}(H)$ is usually easy to check. For example, this is done for the pair $(G, H)=(\mathrm{SO}(n+1) \times \mathrm{SO}(n), \mathrm{SO}(n))$ of algebraic groups over a local field [2, Proposition 1.1].

\section{Proof of Theorem 1.2}

Fix a $K$-invariant positive definite quadratic form $Q$ on $\mathfrak{k}$. Let $X_{1}, X_{2}, \ldots, X_{n}$ be a basis for $\mathfrak{k}$ orthonormal with respect to $Q$. Put

$$
\Omega=\Omega_{Q}=1-\sum_{i=1}^{n} X_{i}^{2} .
$$

Then it is in $\mathrm{U}\left(\mathfrak{k}_{\mathbb{C}}\right)^{K}$ and is independent of the orthonormal basis.

Denote by $\widehat{K}$ the set of equivalence classes of irreducible finite dimensional unitary representations of $K$. For every $\tau \in \widehat{K}$, fix an irreducible unitary representation $\left(\pi_{\tau}, V_{\tau}\right)$ of $K$, of class $\tau$. By Schur's Lemma, $\pi_{\tau}(\Omega)$ acts on $V_{\tau}$ via a scalar $c(\tau)$. It is known and also easy to see that $c(\tau)$ is real and $\geq 1$. Set $d(\tau)=\operatorname{dim} V_{\tau}$.

Lemma 2.1. If $m$ is large enough, then

$$
\sum_{\tau \in \widehat{K}} d(\tau) c(\tau)^{-m}<+\infty
$$

Proof. Lemma 4.4.2.3 of [4] says that

$$
\sum_{\tau \in \widehat{K}} d(\tau)^{2} c(\tau)^{-m}<+\infty
$$

if $m$ is large enough. This implies the lemma.

Now let $m_{0}$ be the smallest nonnegative integer such that (2.1) holds. Set

$$
X=\left(\sum_{\tau \in \widehat{K}} d(\tau) c(\tau)^{-m_{0}}\right) \Omega^{m_{0}},
$$

which is an element of $\mathrm{U}\left(\mathfrak{k}_{\mathbb{C}}\right)^{K}$.

Recall that $(\pi, V)$ is a unitary representation of $G$, and $V^{\infty}$ is the space of smooth vectors. We have a Hilbert space decomposition

$$
V=\widehat{\bigoplus}_{\tau \in \widehat{K}} V(\tau)
$$

where $V(\tau)$ is the $\tau$-isotypic component of $V$, which is automatically closed in $V$. Denote by $P(\tau)$ the orthogonal projection of $V$ onto $V(\tau)$. It maps $V^{\infty}$ into itself. 
By [4, Theorem 4.4.2.1], which is due to Harish-Chandra, we have

Lemma 2.2. If $v \in V^{\infty}$, then the Fourier series

$$
\sum_{\tau \in \widehat{K}} P(\tau) v
$$

converges absolutely to $v$ in $V^{\infty}$.

The following is a variation of Lemma 4.4.2.2 of [4].

Lemma 2.3. For any integer $m \geq 0$, and $v \in V^{\infty}$, one has that

$$
\|P(\tau) v\| \leq c(\tau)^{-m}\left\|\pi\left(\Omega^{m}\right) v\right\| .
$$

Proof. Let $v \in V^{\infty}$. Since $P(\tau) v \in V(\tau) \cap V^{\infty}$, we have that

$$
\pi\left(\Omega^{m}\right)(P(\tau) v)=c(\tau)^{m} P(\tau) v .
$$

Notice that $P(\tau)$ and $\pi\left(\Omega^{m}\right)$ are two commuting elements in $\operatorname{End}\left(V^{\infty}\right)$. Therefore

$$
\begin{aligned}
& \|P(\tau) v\| \\
= & \left\|c(\tau)^{-m} \pi\left(\Omega^{m}\right)(P(\tau) v)\right\| \quad \text { by (2.4) } \\
= & c(\tau)^{-m}\left\|P(\tau)\left(\pi\left(\Omega^{m}\right) v\right)\right\| \\
\leq & c(\tau)^{-m}\left\|\pi\left(\Omega^{m}\right) v\right\| \quad \text { since } P(\tau) \text { is an orthogonal projection. }
\end{aligned}
$$

Lemma 2.4. Let $X$ be as in (2.3). If $v \in V^{\infty}$, then

$$
\left.\sum_{\tau \in \widehat{K}} d(\tau) \| P(\tau) v\right)|| \leq|v|_{X} .
$$

Proof. We have

$$
\begin{aligned}
& \left.\sum_{\tau \in \widehat{K}} d(\tau) \| P(\tau) v\right) \| \\
\leq & \sum_{\tau \in \widehat{K}} d(\tau) c(\tau)^{-m_{0}}\left\|\pi\left(\Omega^{m_{0}}\right) v\right\| \quad \text { by Lemma } 2.3 \\
= & \|\pi(X) v\| \quad \text { by (2.3) } \\
= & |v|_{X} .
\end{aligned}
$$

Now we are ready to prove Theorem 1.2. Assume that $\pi$ is tempered. Let $g \in G$, $u \in V^{\infty}$ and $v \in V$. Then

$$
\begin{aligned}
& |\langle\pi(g) u, v\rangle| \\
= & \left|\left\langle\pi(g)\left(\sum_{\tau \in \widehat{K}} P(\tau) u\right), v\right\rangle\right| \quad \text { by Lemma 2.2 } \\
\leq & \sum_{\tau \in \widehat{K}}|\langle\pi(g)(P(\tau) u), v\rangle| .
\end{aligned}
$$

As a part of [1, Theorem 2], it is known and also easy to see that

$$
d_{P(\tau) u} \leq d(\tau)^{2} .
$$


Let $X$ be as in (2.3). If $v$ is $K$-finite, then

$$
\begin{aligned}
& |\langle\pi(g) u, v\rangle| \\
\leq & \left.\sum_{\tau \in \widehat{K}} d(\tau) d_{v}^{\frac{1}{2}} \| P(\tau) u\right)\|\| v \| \Xi(g) \quad \text { by (2.5), (2.6) and Theorem 1.1] } \\
\leq & d_{v}^{\frac{1}{2}}|u|_{X}\|v\| \Xi(g) \quad \text { by Lemma 2.4. }
\end{aligned}
$$

This proves (1.3) of the theorem.

Now assume that $v \in V^{\infty}$. Then

$$
\begin{aligned}
& |\langle\pi(g) u, v\rangle| \\
\leq & \sum_{\tau \in \widehat{K}}\left|\left\langle\pi(g)(P(\tau) u), \sum_{\tau^{\prime} \in \widehat{K}} P\left(\tau^{\prime}\right) v\right\rangle\right| \quad \text { by (2.5) and Lemma 2.2 } \\
\leq & \sum_{\tau \in \widehat{K}} \sum_{\tau^{\prime} \in \widehat{K}}\left|\left\langle\pi(g)(P(\tau) u), P\left(\tau^{\prime}\right) v\right\rangle\right| \\
\leq & \sum_{\tau \in \widehat{K}} \sum_{\tau^{\prime} \in \widehat{K}} d(\tau) d\left(\tau^{\prime}\right)\|P(\tau) u\|\left\|P\left(\tau^{\prime}\right) v\right\| \Xi(g) \quad \text { by (2.6) and Theorem 1.1 } \\
\leq & |u|_{X}|v|_{X} \Xi(g) \quad \text { by Lemma 2.4. }
\end{aligned}
$$

This proves (1.2) of the theorem.

\section{REFERENCES}

1. M. Cowling, U. Haagerup and R. Howe, Almost $L^{2}$ matrix coefficients. J. Reine Angew. Math. 387 (1988), 97-110. MR946351 (89i:22008)

2. A. Ichino and T. Ikeda, On the periods of automorphic forms on special orthogonal groups and the Gross-Prasad conjecture, preprint.

3. N. Wallach, Real Reductive Groups I, Academic Press, San Diego, 1988. MR.929683 (89i:22029)

4. G. Warner, Harmonic Analysis on Semi-simple Lie Groups I, Springer-Verlag, New York, 1972. MR0498999(58:16979)

Academy of Mathematics and Systems Science, Chinese Academy of Sciences, Beijing, 100190, People's Republic of China

E-mail address: sun@math.ac.cn 